\title{
Quantitative proteomic analysis of Down syndrome in the umbilical cord blood using iTRAQ
}

\author{
WEIGUO SUI $^{1 *}$, RUOHAN ZHANG $^{1,2^{*}}$, JIEJING CHEN $^{1}$, HUIYAN HE $^{1,2}$, ZHENZHEN CUI $^{1,2}$,

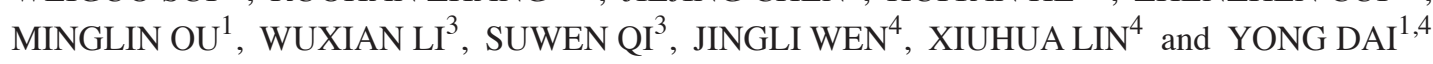 \\ ${ }^{1}$ Nephrology Department of Guilin 181st Hospital, Guangxi Key Laboratory of Metabolic Diseases Research, Guilin, \\ Guangxi 541002; ${ }^{2}$ College of Life Science, Guangxi Normal University, Guilin, Guangxi 541004; \\ ${ }^{3}$ Key Laboratory of Laboratory Medical Diagnostics of Education Ministry, Chongqing Medical University, \\ Chongqing, Sichuan 400016; ${ }^{4}$ Clinical Medical Research Center, The Second Clinical Medical College \\ of Jinan University (Shenzhen People's Hospital), Shenzhen, Guangdong 518020, P.R. China
}

Received October 16, 2013; Accepted May 22, 2014

DOI: $10.3892 / \mathrm{mmr} .2014 .2828$

\begin{abstract}
Down sydrome (DS) is a relatively frequent chromosomal disorder, which has no safe and effective method of prenatal diagnosis to date. The present study was designed to identify DS biomarkers. We quantified the changes in the umbilical cord blood protein levels between DS-affected and healthy (control) pregnant females using isobaric tags for relative and absolute quantification (iTRAQ) and Gene Ontology (GO) analysis. A total of 505 proteins were identified, and of these, five proteins showed significantly different concentrations between the DS and the control group. These proteins may thus be relevant to DS and constitute potential DS biomarkers.
\end{abstract}

\section{Introduction}

Down sydrome (DS) is a relatively frequent chromosomal disorder. It is diagnosed in 1:500 to 1:800 of pregnant females (1). DS is clinically manifested by multiple somatic anomalies, mental retardation and precocious dementia. Pathological examination of DS brains reveals poor maturation, atrophy of the dendrites and the early appearance of senile plaques, which are characteristic of Alzheimer's disease (AD). DS represents an important issue for affected families and the society, but there is no effective treatment at

Correspondence to: Dr Yong Dai, Clinical Medical Research Center, The Second Clinical Medical College of Jinan University (Shenzhen People's Hospital), 1017 Dongmen North Road, Shenzhen, Guangdong 518020, P.R. China

E-mail: daiyong2222@gmail.com

*Contributed equally

Key words: Down syndrome, proteomics, isobaric tags for relative and absolute quantification, Gene Ontology analysis present. Advanced maternal age (35 years old) is an important risk factor for fetal DS (2). The most popular diagnostic strategy for DS relies in the combined detection of AFP, hCG, $\mathrm{uE} 3$ and inhibin $\mathrm{A}$, with a detection rate of $60-75 \%$ and a $5 \%$ false positive rate (3). It is important to find a safe and effective method for diagnosis of DS. One strategy to achieve this is via identification of new biomarkers using noninvasive proteomic approaches. In a previous study, the isobaric tags for relative and absolute quantification (iTRAQ) technique was combined with matrix-assisted laser desorption/ionization (MALDI) time of flight (TOF)/TOF select biomarkers (4). In this study, iTRAQ was combined with Gene Ontology (GO) analysis, in order to identify the proteins that are differentially expressed in DS and their predicted functions.

Proteomic approaches are a promising tool for the identification of diagnostic biomarkers of DS (5). iTRAQ was developed by Applied Biosystems, Inc. (Foster City, CA, USA) in 2004. iTRAQ-based proteomic analysis represents a major development in the rapid detection of potential biomarkers. The key advantage of the 8-plex iTRAQ system is the ability to simultaneously analyze up to 8 different biological specimens, thereby increasing the throughput while reducing experimental errors (6). iTRAQ has been recently employed in the study of certain diseases (7-9). In this study, we used iTRAQ in conjunction with multidimensional chromatography, followed by GO analysis, to detect and quantify proteome differences.

\section{Materials and methods}

Samples. Umbilical cord blood samples were obtained from April-August 2011 from pregnant females, six carrying a DS fetus (age, 27-37) and 11 carrying a healthy fetus (age, 27-37), at the Shenzhen People's Hospital (Shenzhen, China). Diagnosis of DS in these women was performed by chromosomal examination. This study was undertaken with the approval of the Institutional Ethical Board of Guilin 181st Hospital (Guilin, China), and written informed consent was provided by all subjects. 
Table I. The upregulated and downregulated proteins in umbilical cord blood samples of Down syndrome fetuses.

\begin{tabular}{|c|c|c|c|}
\hline UniProt accession no. & Protein name & Peptides & Ratio \\
\hline \multicolumn{4}{|c|}{ Upregulated proteins $(n=13)$} \\
\hline Q8N1G4 & Leucine-rich repeat-containing protein 47 & 3 & 60.27 \\
\hline Q9UNM6 & $26 \mathrm{~S}$ proteasome non-ATPase regulatory subunit 13 & 1 & 60.27 \\
\hline P55209 & Nucleosome assembly protein 1-like 1 & 2 & 60.27 \\
\hline Q6P1M3 & Lethal (2) giant larvae protein homolog 2 & 1 & 60.27 \\
\hline Q96HE7 & ERO1-like protein $\alpha$ & 2 & 60.27 \\
\hline Q9ULD0 & 2-oxoglutarate dehydrogenase-like, mitochondrial & 10 & 60.27 \\
\hline P63313 & Thymosin $\beta-10$ & 4 & 60.27 \\
\hline O43681 & ATPase ASNA1 & 3 & 60.27 \\
\hline Q9UHI5 & Large neutral amino acids transporter small subunit 2 & 2 & 60.27 \\
\hline Q9Y6X5 & Ectonucleotide pyrophosphatase/phosphodiesterase family member 4 & 1 & 60.27 \\
\hline P02649 & Apolipoprotein E & 40 & 3.75 \\
\hline $\mathrm{P} 00751$ & Complement factor B & 7 & 1.96 \\
\hline $\mathrm{P} 02743$ & Amyloid P component, serum & 7 & 1.68 \\
\hline \multicolumn{4}{|c|}{ Downregulated proteins $(n=6)$} \\
\hline Q9H1E5 & Thioredoxin-related transmembrane protein 4 & 1 & 0.01 \\
\hline Q9UHV9 & Prefoldin subunit 2 & 3 & 0.01 \\
\hline Q01433 & AMP deaminase 2 & 1 & 0.01 \\
\hline Q00765 & Receptor expression-enhancing protein 5 & 4 & 0.01 \\
\hline P43243 & Matrin-3 & 4 & 0.01 \\
\hline P10451 & Osteopontin & 1 & 0.44 \\
\hline
\end{tabular}

Ratio, the ratio of the ionic strength of the DS group and the ionic strength of the control group. Proteins that provided tryptic peptides with an average reporter ion ratio $\geq 1.5$ were classified as upregulated and those with an average reporter ion ratio $\leq 0.67$ were classified as downregulated.

Sample preparation. Cord blood $(10 \mathrm{ml})$ was collected from enrolled subjects in heparinized vacutainers. The samples were centrifuged at $3,000 \times \mathrm{g}$ for $30 \mathrm{~min}$ at $10^{\circ} \mathrm{C}$, the plasma was separated, and $100-\mu 1$ aliquots were stored at $-80^{\circ} \mathrm{C}$ until further use.

Protein extraction. Total protein was extracted from the plasma samples with the ProteoMiner protein enrichment kit (Bio-Rad Laboratories, Hercules, CA, USA) as per the manufacturer's instructions. Protein concentration was measured with the Pierce ${ }^{\mathrm{TM}} \mathrm{BCA}$ Protein Assay kit (Thermo Fisher Scientific, Waltham, MA, USA). A previous study indicated that extensive analysis of well-characterized pooled samples is more effective than analysis of individual samples (10). In this study, $40 \mu \mathrm{g}$ of protein from each sample was pooled with protein samples of the DS or control group prior to proteomic analysis.

iTRAQ, strong cation exchange (SCX) and mass spectrometry $(M S) / M S$. The proteins from each pool were blocked, digested, and labeled in accordance with the protocol of the Applied Biosystems iTRAQ ${ }^{\mathrm{TM}}$ Reagents system (Thermo Fisher Scientific). The iTRAQ tags were as follows: healthy control, 114; DS, 116. The labeled peptides were combined into one sample.

Multidimensional liquid chromatography was performed to separate the tryptic peptides prior to MS. The combined samples were separated into $10 \mathrm{SCX}$ fractions using a

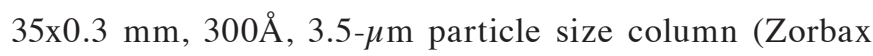
Bio-SCX; Agilent, Santa Clara, CA, USA) with a potassium formate gradient in $25 \%$ acetonitrile. The peptides in these fractions were further separated on a Tempo ${ }^{\mathrm{TM}} \mathrm{LC}$ nanoflow and MALDI spotting system, equipped with a reversed-phase Magic C18AQ column (Applied Biosystems Life Technologies, Foster City, CA, USA). Each chromatography run yielded 380 MALDI spots on a stainless steel MALDI target plate, by the same method as previously described (4).

MS data acquisition was conducted with a 4800 Plus MALDI TOF/TOF ${ }^{\mathrm{TM}}$ analyzer (Applied Biosystems, Inc.). Only peaks with a signal-to-noise ratio $\geq 40$ were selected for tandem mass spectrometry. Mass spectra from 500 laser shots were acquired for each spot. The combined MS/MS data from all 10 fractions were used for a Paragon Algorithm (11) search. Human version 3.62 proteome data were downloaded from the EBI website (http://www.ebi.ac.uk/) (4).

Statistical and GO analysis. Proteins that provided tryptic peptides with an average reporter ion ratio $\geq 1.5$ were classified as upregulated and those with an average reporter ion ratio $\leq 0.67$ as downregulated. To further investigate the functions of the identified proteins, we used the online GO tool WEGO (http://wego.genomics.org.cn/), which allowed annotation of the proteins with regards to the molecular function (MF), cellular component (CC) and biological process (BP) with which they are associated. 


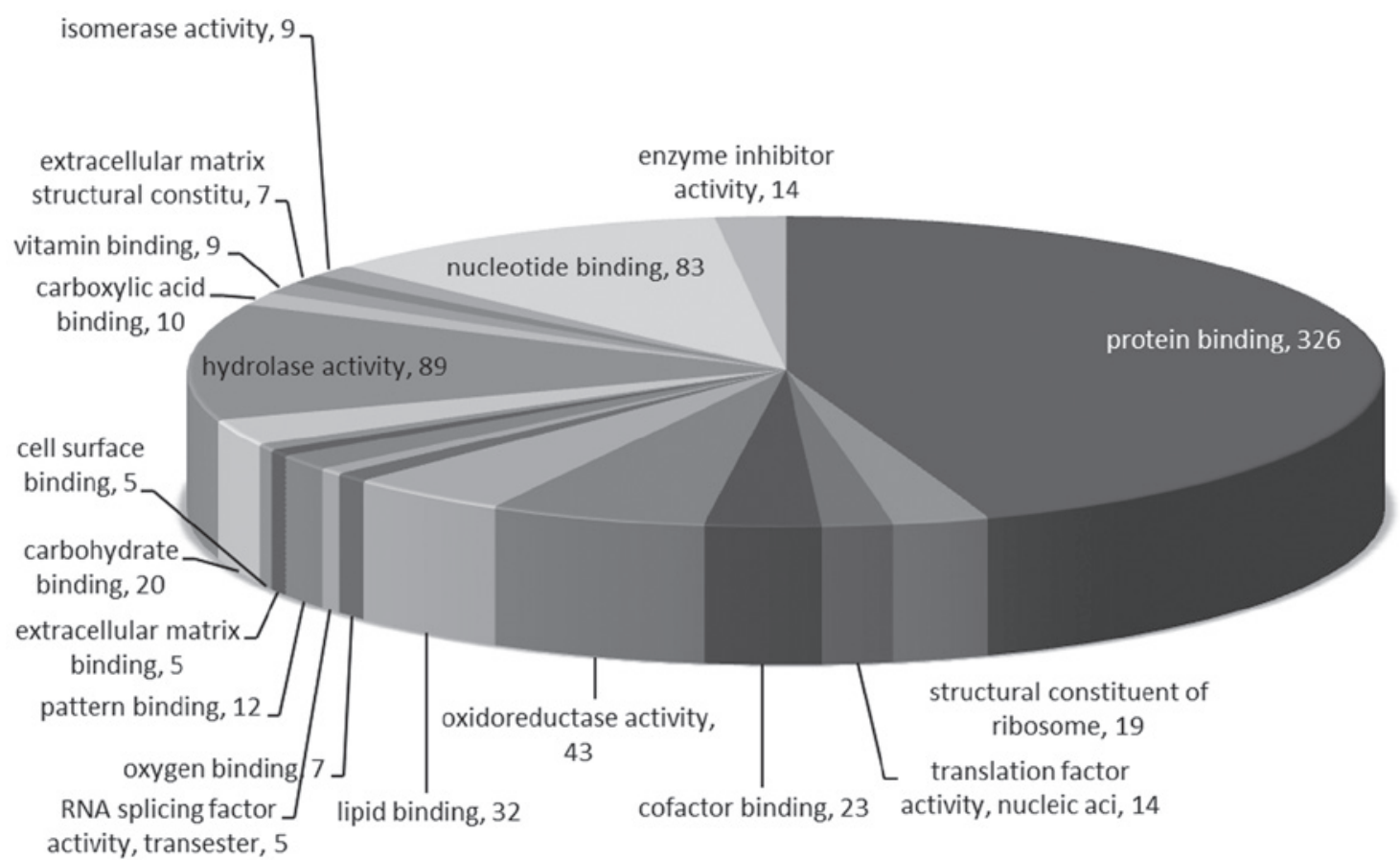

Figure 1. Classification of the 505 plasma proteins in different molecular function categories based on Gene Ontology terms.

Table II. The top 5 molecular function, cellular component and biological process Gene Ontology terms for the 505 proteins.

A, Top 5 molecular functions

\begin{tabular}{lrc}
\hline Molecular function & Count & Proportion (\%) \\
\hline Protein binding & 326 & 64.94 \\
Hydrolase activity & 89 & 17.73 \\
Nucleotide binding & 83 & 16.53 \\
Oxidoreductase activity & 43 & 8.57 \\
Lipid binding & 32 & 6.37 \\
\hline
\end{tabular}

B, Top 5 cellular components

\begin{tabular}{lcc}
\hline Cellular component & Count & Proportion (\%) \\
\hline Intracellular & 405 & 80.68 \\
Intracellular part & 403 & 80.28 \\
Intracellular organelle & 318 & 63.35 \\
Membrane-bounded organelle & 278 & 55.38 \\
Organelle part & 191 & 38.05 \\
\hline
\end{tabular}

\section{C, Top 5 biological processes}

\begin{tabular}{lcc}
\hline Biological process & Count & Proportion (\%) \\
\hline Primary metabolic process & 244 & 48.61 \\
Cellular metabolic process & 230 & 45.82 \\
Establishment of localization & 124 & 24.7 \\
Transport & 123 & 24.5 \\
Response to stress & 91 & 18.13 \\
\hline
\end{tabular}

\section{Results}

Proteome of the umbilical cord blood. Using peptides of $>1$ an average reporter ion ratio $\geq 1.5$ and an average reporter ion ratio $\leq 0.67$ as cutoffs, 505 proteins were identified and quantified from the plasma. These proteins, from the pooled sample composed of individuals diagnosed with DS, were divided into 13 upregulated and 6 downregulated compared to healthy subjects, as shown in Table I.

Among the differentially expressed proteins in Table I and based on published studies, 5 proteins [apolipoprotein $\mathrm{E}$, complement factor $\mathrm{B}(\mathrm{CFB})$, amyloid $\mathrm{P}$ component, serum (APCS), matrin-3 and osteopontin (OPN)] were found to be relevant to DS, with the first three being markedly upregulated in DS. The remaining proteins [leucine-rich repeat-containing protein $47,26 \mathrm{~S}$ proteasome non-ATPase regulatory subunit 13 , nucleosome assembly protein 1-like 1 , lethal (2) giant larvae protein homolog 2, endoplasmic oxidoreductin-1-like (ERO1-like) protein $\alpha, 2$-oxoglutarate dehydrogenase-like, mitochondrial, thymosin $\beta$-10, ATPase ASNA1, large neutral amino acids transporter small subunit 2, ectonucleotide pyrophosphatase/phosphodiesterase family member 4, thioredoxin-related transmembrane protein 4, prefoldin subunit 2, AMP deaminase 2 and receptor expression-enhancing protein 5] showed the highest ratios (60.27 and $0.01)$, compared to healthy controls.

The 505 proteins were assigned to MF, CC and BP GO terms; the distribution of these proteins in the three GO term categories is shown in Figs. 1-3. The top 5 MFs in terms of relative proportion were protein binding, hydrolase activity, nucleotide binding, oxidoreductase activity and lipid binding (Table IIA). The top 5 CCs were intracellular, intracellular part, intracellular organelle, membrane-bounded organelle and organelle part (Table IIB). The top 5 BPs were primary metabolic process, cellular metabolic process, estab- 


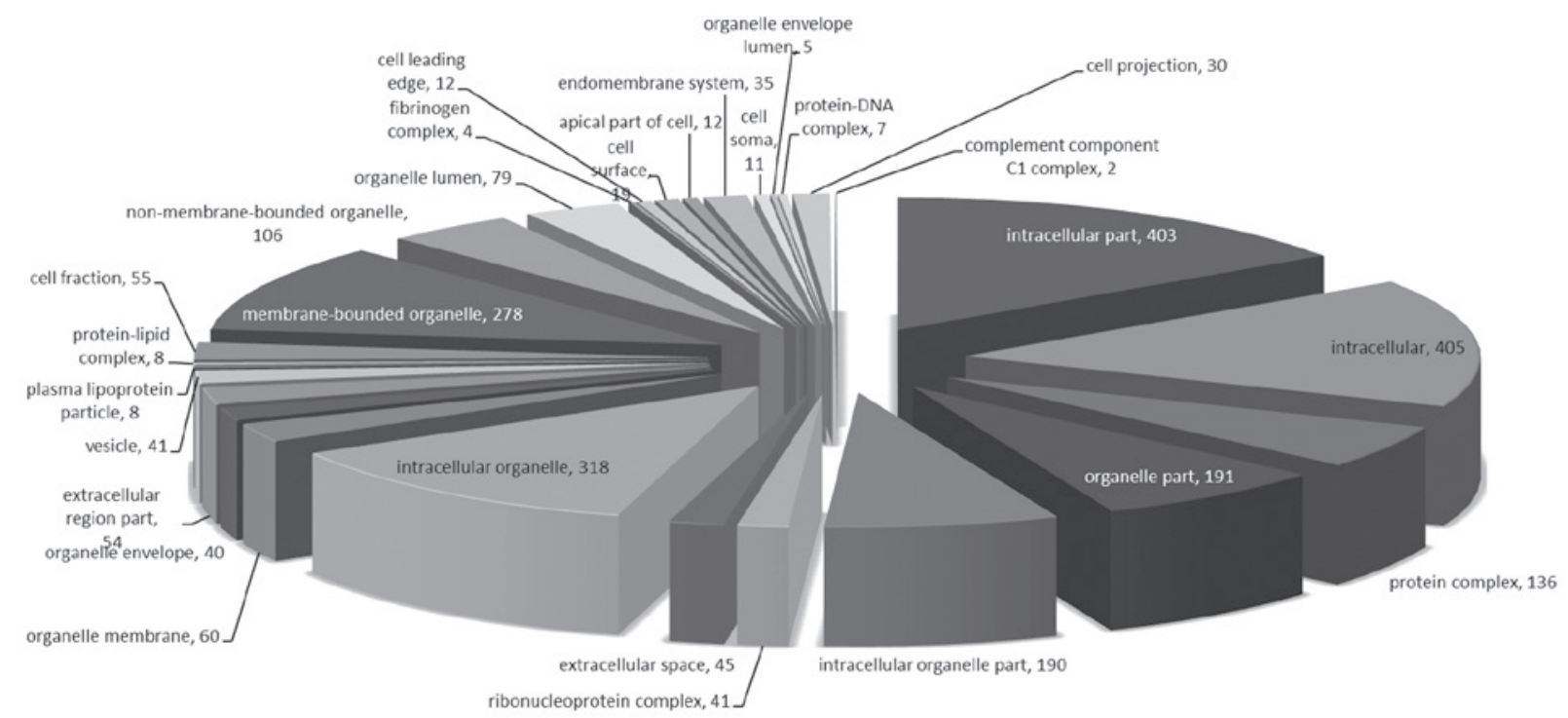

Figure 2. Classification of the 505 plasma proteins in different cellular component categories based on Gene Ontology terms.

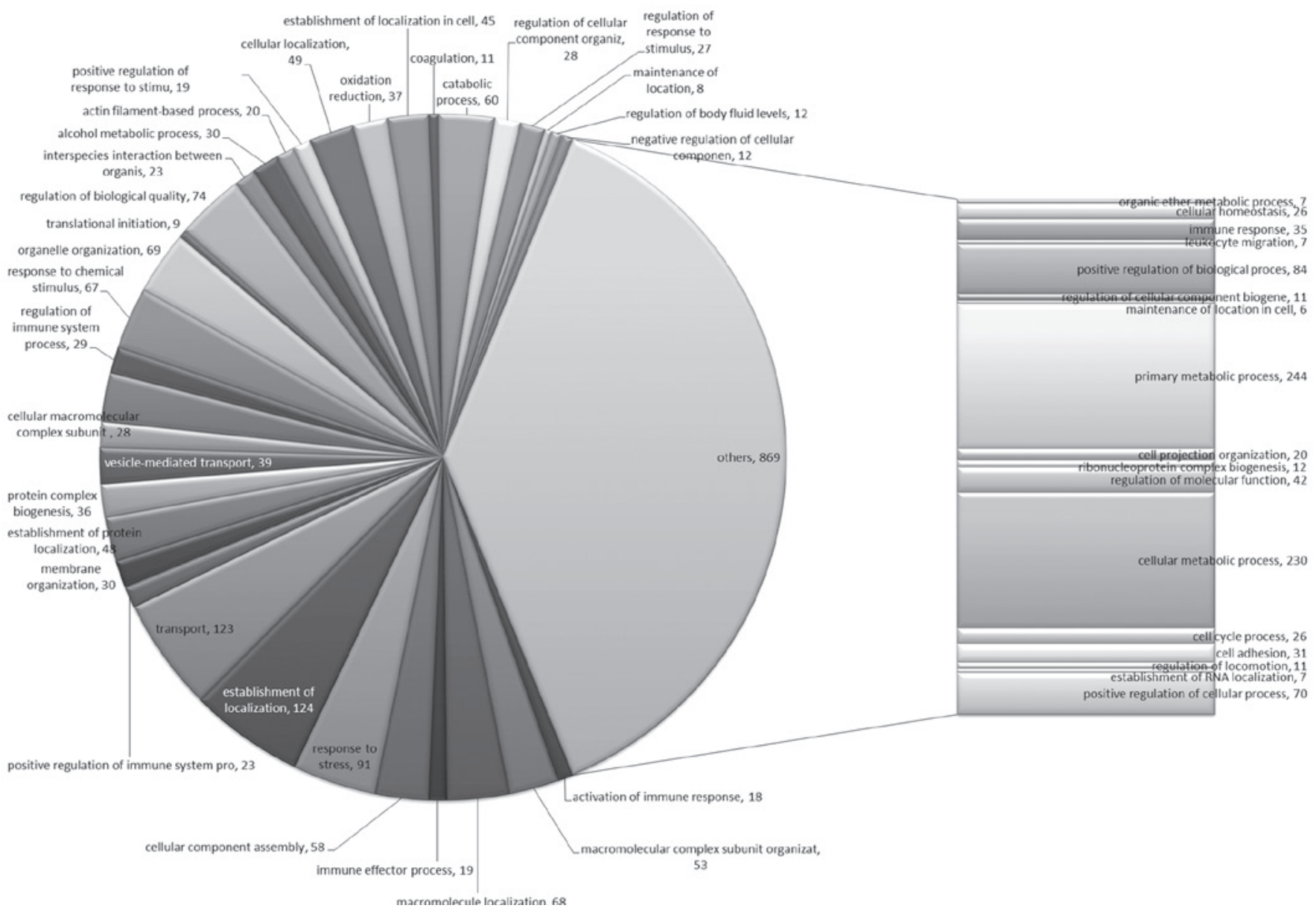

Figure 3. Classification of the 505 plasma proteins in different biological process categories based on Gene Ontology terms.

lishment of localization, transport and positive regulation of BP (Table IIC). The full list of MF, CC and BP associated with apolipoprotein E, CFB, APCS, matrin-3 and OPN is shown in Table III. MF, CC and BP terms for the proteins which showed the highest fold difference are presented in Table IV.

\section{Discussion}

Quantification of the proteins via iTRAQ analysis has been suggested to be a suitable strategy for the identification of biomarkers, since iTRAQ allows the comparison of protein 


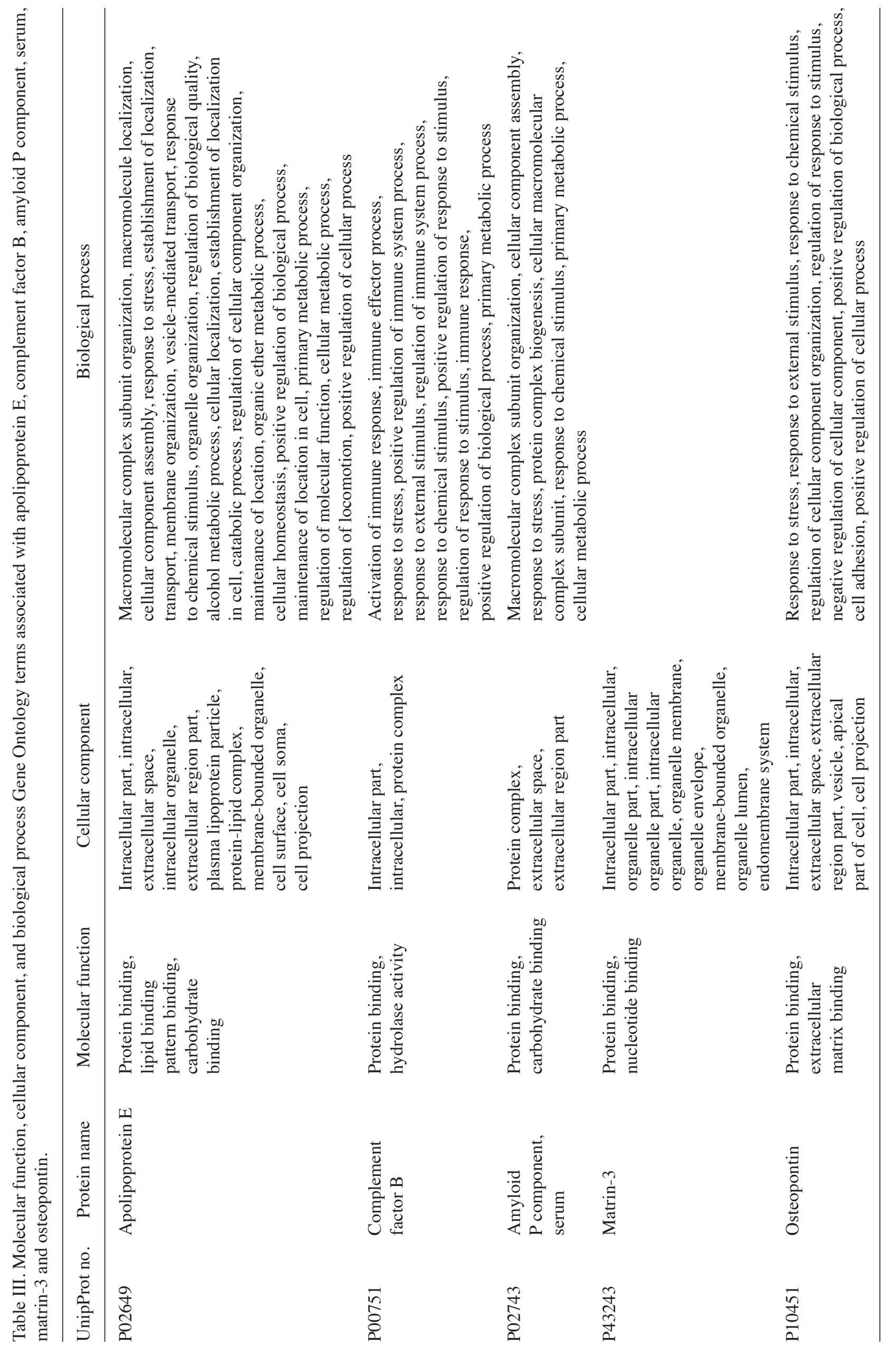




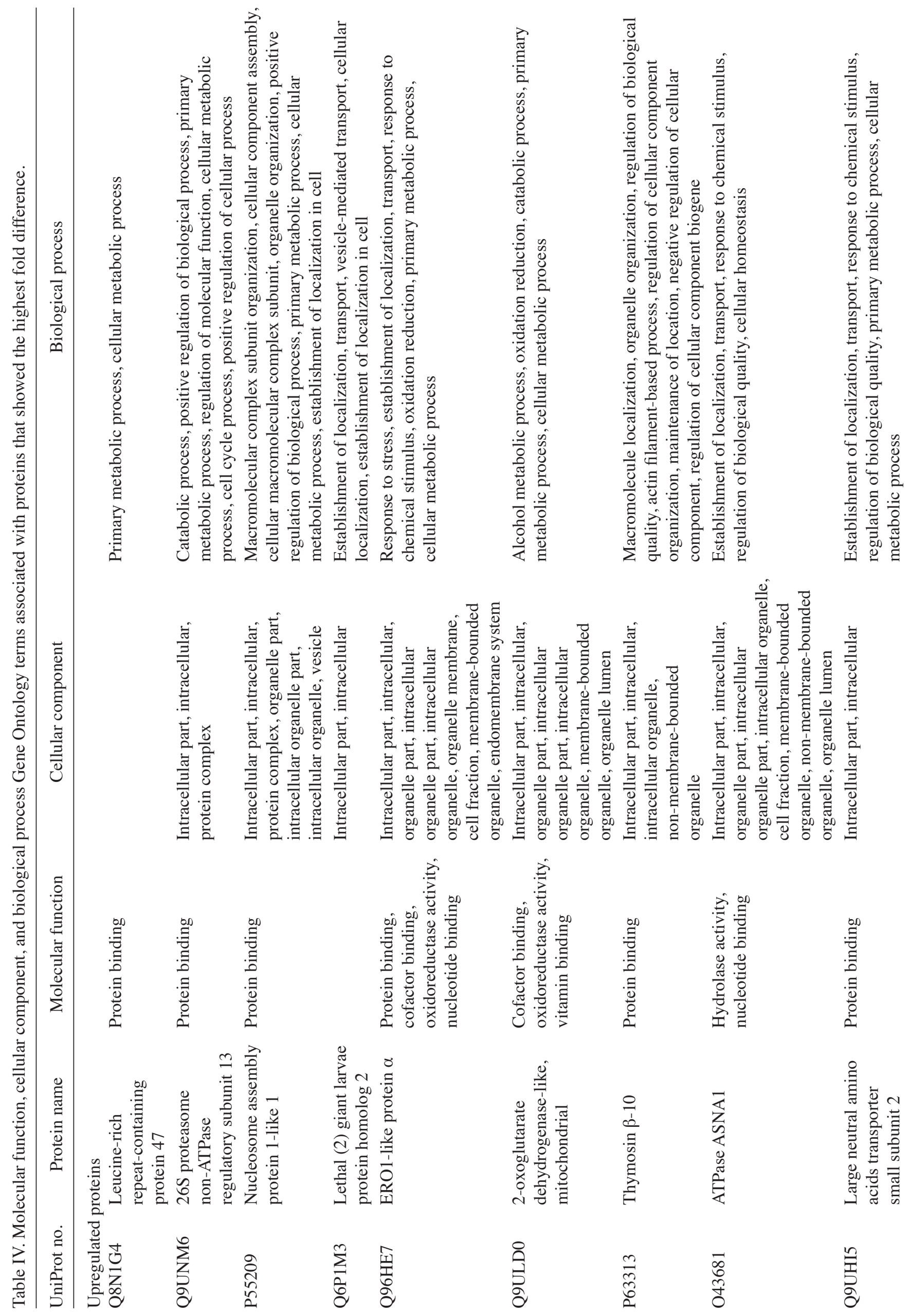


abundance among samples by measuring the peak intensities of reporter ions released from the iTRAQ-tagged peptides. In this study, we adopted iTRAQ technology and GO analysis to quantitatively analyse the proteome of plasma from the umbilical cord blood of DS fetus-carrying mothers, in order to identify useful biomarkers for DS. As a result, 505 proteins were identified, and were classified to MF, CC and BP terms by $\mathrm{GO}$ analysis. Among the up- and downregulated proteins in DS (Table I), we focused on 5 (apolipoprotein E, CFB, APCS, matrin-3, and OPN) to verify whether these may constitute novel DS biomarkers.

A putative risk factor for $\mathrm{AD}$ in the general population, the $\mathrm{E} 4$ allele of the apolipoprotein $\mathrm{E}$ gene, has highlighted the role of genetic influences in AD. It has long been recognized that DS is associated with early and severe development of AD neuropathological lesions (12). APCS and the Ig $\lambda$ chain C region were also found in the sera of patients with DS in another study (8). We found that the concentration of apolipoprotein $\mathrm{E}$ in the plasma of umbilical cord blood was 3.75 times (x) higher in the DS compared to the control group The MF, $\mathrm{CC}$ and BP GO terms associated with apolipoprotein $\mathrm{E}$ are shown in Table III.

CFB plays an important role in the alternative complement pathway in AD (13), which shares a number of similar features with DS. Yu et al (14) found that the concentration of CFB is significantly increased in the serum of mothers with fetuses affected by DS. CFB may be associated with the brain damage that occurs in DS. In certain studies, CFB was associated with brain diseases, and a relationship between DS and the complement system was revealed (13,15-17). In the present study, we found that the concentration of CFB in the plasma of umbilical cord blood was $1.95 \mathrm{x}$ higher in the DS compared to the control group. The MFs associated with CFB were protein binding and hydrolase activity. The CCs associated with $\mathrm{CFB}$ were intracellular part, intracellular and protein complex. The BPs associated with CFB included activation of immune response, immune effector process, and response to stress (full list in Table III).

The APCS glycoprotein is encoded by a single gene locating on the human chromosome 1, has 204 residues, and is secreted (18). APCS immunoreactivity is commonly observed in DS, AD, and amyloid disorders with primarily cerebrovascular compromise $(19,20)$. In the present study, we found that the concentration of APCS in the plasma of umbilical cord blood was $1.68 \mathrm{x}$ higher in the DS compared to the control group. The MFs, CCs and BPs associated with APCS are listed in Table III. The MFs were protein and carbohydrate binding, the CCs were protein complex, extracellular space and extracellular region part, and the BPs included macromolecular complex subunit organization, CC assembly, response to stress, and protein complex biogenesis (Table III).

OPN is a secreted glycoprotein with an arginine-glycine-aspartic acid (RGD) sequence, and able to bind a number of receptors, including the integrins and certain variant forms of CD44. OPN plays an important role in diverse fibro-inflammatory diseases. OPN is expressed in hepatocytes and macrophage-like cells in DS individuals showing perinatal liver fibrosis. OPN was thus proposed to be involved in the pathogenesis of perisinusoidal liver fibrosis, frequently observed in neonates with DS (21). We found that the concen- 
tration of OPN in the plasma of umbilical cord blood was $2.27 \mathrm{x}$ lower in the DS compared to the control group. The MFs, CCs and BPs associated with OPN are displayed in Table III. The MFs were protein and extracellular matrix binding, the $\mathrm{CCs}$ included intracellular part, intracellular, and extracellular space, while the BPs included response to stress, external stimulus, and chemical stimulus (Table III).

Matrin-3 is an inner nuclear matrix protein of $125 \mathrm{kDa}$, which is highly conserved in mammals (22). Matrin-3 contains a bipartite nuclear localization signal, two zinc finger domains, and two canonical RNA recognition motifs (23). Matrin-3 is encoded by the MATR 3 gene, which is expressed in skeletal muscle (24). Autosomal and the active $\mathrm{X}$ chromosome territories were found to express matrin-3 (25). Matrin-3 was also found to be a target of the ataxia telangiectasia mutated (ATM) protein kinase (26). Degradation of matrin-3 may be a key cellular event, which induces a shift from apoptotic to necrotic death (27). Matrin-3 expression was significantly decreased in the fetal DS brain (28). The manifold decreased spot unambiguously assigned to matrin-3 in previous studies may reflect or induce aberrant transcription reported to occur in DS $(28,29)$. In this study, we found that the concentration of matrin-3 in the plasma of umbilical cord blood was 100x lower in the DS compared to the control group. We did not found a BP term associated with matrin-3. The MFs associated with matrin-3 were protein and nucleotide binding, and the CCs included intracellular part, intracellular, and organelle part (Table III).

In conclusion, the iTRAQ technology, a relatively new strategy for proteomic analysis, was used here to study the changes in protein expression associated with DS. This approach identified 5 significantly differentially expressed proteins (apolipoprotein E, CFB, APCS, matrin-3 and OPN), which were previously reported to relate to DS. The MFs, CCs and BPs associated with these proteins indicate that they may be suitable DS biomarkers. However, further investigation of the functions of these proteins, as well as of the proteins with high fold changes in DS identified herein is required.

\section{Acknowledgements}

We thank the patients and healthy volunteers who participated in this study, as well as teachers and classmates for their help. This study was supported by the Guangxi Key Laboratory Construction Project planning program 12-071-32, the Guangdong Provincial S\&T program (2012B032000008), the Shenzhen S\&T program 201202121 (2013-2014), the Innovation and Technology Commission of the Shenzhen Municipal Science and Technology Plan Project (CXZZ20130321090846345) and the Guilin Technology Plan (20110328 and 20130120-21).

\section{References}

1. Sherman SL, Allen EG, Bean LH and Freeman SB: Epidemiology of Down syndrome. Ment Retard Dev Disabil Res Rev 13: 221-227, 2007.

2. Kwon JY, Park IY, Kwon SM, Kim CJ and Shin JC: The quadruple test for Down syndrome screening in pregnant women of advanced maternal age. Arch Gynecol Obstet 285: 629-633, 2012.
3. Kolialexi A, Tsangaris GT, Papantoniou N, et al: Application of proteomics for the identification of differentially expressed protein markers for Down syndrome in maternal plasma. Prenat Diagn 28: 691-698, 2008.

4. Wang L, Dai Y, Qi S, Sun B, Wen J, Zhang L and Tu Z: Comparative proteome analysis of peripheral blood mononuclear cells in systemic lupus erythematosus with iTRAQ quantitative proteomics. Rheumatol Int 32: 585-593, 2012.

5. Garbis SD, Tyritzis SI, Roumeliotis T, et al: Search for potential markers for prostate cancer diagnosis, prognosis and treatment in clinical tissue specimens using amine-specific isobaric tagging (iTRAQ) with two-dimensional liquid chromatography and tandem mass spectrometry. J Proteome Res 7: 3146-3158, 2008.

6. Bouchal P, Roumeliotis T, Hrstka R, Nenutil R, Vojtesek B and Garbis SD: Biomarker discovery in low-grade breast cancer using isobaric stable isotope tags and two-dimensional liquid chromatography-tandem mass spectrometry (iTRAQ-2DLC-MS/MS) based quantitative proteomic analysis. J Proteome Res 8: 362-373, 2009.

7. Sun C, Song C, Ma Z, et al: Periostin identified as a potential biomarker of prostate cancer by iTRAQ-proteomics analysis of prostate biopsy. Proteome Science 9: 22, 2011.

8. Kolla V, Jenö P, Moes S, Tercanli S, Lapaire O, Choolani M and Hahn S: Quantitative proteomics analysis of maternal plasma in Down syndrome pregnancies using isobaric tagging reagent (iTRAQ). J Biomed Biotechnol 2010: 952047, 2010.

9. Sui W, Tang D, Zou G, Chen J, Ou M, Zhang Y and Dai Y: Differential proteomic analysis of renal tissue in lupus nephritis using iTRAQ reagent technology. Rheumatol 32: 3537-3543, 2012 .

10. Abdi F, Quinn JF, Jankovic J, et al: Detection of biomarkers with a multiplex quantitative proteomic platform in cerebrospinal fluid of patients with neurodegenerative disorders. J Alzheimers Dis 9: 293-348, 2006.

11. Shilov IV, Seymour SL, Patel AA et al. The Paragon Algorithm, a next generation search engine that uses sequence temperature values and feature probabilities to identify peptides from tandem mass spectra. Mol Cell Proteomics 6: 1638-1655, 2007.

12. Hyman BT, West HL, Rebeck GW, et al: Quantitative analysis of senile plaques in Alzheimer disease: Observation of log-normal size distribution and molecular epidemiology of differences associated with apolipoprotein E genotype and trisomy 21 (Down syndrome). Proc Natl Acad Sci USA 92: 3586-3590, 1995.

13. Strohmeyer R, Shen Y and Rogers J: Detection of complement alternative pathway mRNA and proteins in the Alzheimer's disease brain. Brain Res Mol Brain Res 81: 7-18, 2000.

14. Yu B, Zhang B, Wang J, Wang QW, Huang RP, Yang YQ and Shao SH: Preliminary proteomic-based identification of a novel protein for Down's syndrome in maternal serum. Exp Biol Med (Maywood) 237: 530-539, 2012.

15. Kossmann T, Stahel PF, Morganti-Kossmann MC, Jones JL and Barnum SR: Elevated levels of the complement components C3 and factor B in ventricular cerebrospinal fluid of patients with traumatic brain injury. J Neuroimmunol 73: 63-69, 1997.

16. Head E, Azizeh BY, Lott IT, Tenner AJ, Cotman CW and Cribbs DH: Complement association with neurons and beta-amyloid deposition in the brains of aged individuals with Down syndrome. Neurobiol Dis 8: 252-265, 2001.

17. Stoltzner SE, Grenfell TJ, Mori C, Wisniewski KE, Wisniewski TM, Selkoe DJ and Lemere CA: Temporal accrual of complement proteins in amyloid plaques in Down's syndrome with Alzheimer's disease. Am J Pathol 156: 489-499, 2000.

18. Pepys MB, Baltz ML, de Beer FC, et al: Biology of serum amyloid P component. Ann NY Acad Sci 389: 286-298, 1982.

19. Perlmutter LS, Barrón E, Myers M, Saperia D and Chui HC: Localization of amyloid P component in human brain: vascular staining patterns and association with Alzheimer's disease. J Comp Neurol 352: 92-105, 1995.

20. Rowe IF, Jennson O, Lewis P, Candy J, Tennent GA and Pepys MB: Immunohistochemical demonstration of amyloid $\mathrm{P}$ component in cerebro-vascular amyloidosis. Neuropathol Appl Neurobiol 10: 53-61, 1984.

21. Tokairin T, Nishikawa Y, Watanabe H, et al: Osteopontin expression in the liver with severe perisinusoidal fibrosis: Autopsy case of Down syndrome with transient myeloproliferative disorder. Pathol Int 58: 64-68, 2008.

22. Belgrader P, Dey R and Berezney R: Molecular cloning of matrin 3. A 125-kilodalton protein of the nuclear matrix contains an extensive acidic domain. J Biol Chem 266: 9893-9899, 1991. 
23. Hisada-Ishii S, Ebihara M, Kobayashi N and Kitagawa Y: Bipartite nuclear localization signal of matrin 3 is essential for vertebrate cells. Biochem Biophys Res Commun 354: 72-76, 2007.

24. Senderek J, Garvey SM, Krieger M, et al: Autosomal-dominant distal myopathy associated with a recurrent missense mutation in the gene encoding the nuclear matrix protein, matrin 3. Am J Hum Genet 84: 511-518, 2009.

25. Zeitz MJ, Malyavantham KS, Seifert B and Berezney R: Matrin 3: chromosomal distribution and protein interactions. J Cell Biochem 108: 125-133, 2009.

26. Salton M, Lerenthal Y, Wang SY, Chen DJ and Shiloh Y: Involvement of Matrin 3 and SFPQ/NONO in the DNA damage response. Cell Cycle 9: 1568-1576, 2010.
27. Przygodzka P, Boncela J and Cierniewski CS: Matrin 3 as a key regulator of endothelial cell survival. Exp Cell Res 317: 802-811, 2011.

28. Bernert G1, Fountoulakis M and Lubec G: Manifold decreased protein levels of matrin 3, reduced motor protein HMP and hlark in fetal Down's syndrome brain. Proteomics 2: 1752-1757, 2002.

29. Labudova O, Krapfenbauer K, Moenkemann H, Rink H, Kitzmüller E, Cairns N and Lubec G: Decreased transcription factor junD in brains of patients with Down syndrome. Neurosci Lett 252: 159-162, 1998. 